\title{
Classical Higgs fields on gauge gluon bundles
}

\author{
Marcella Palese ${ }^{1, \star}$ and Ekkehart Winterroth ${ }^{1,2, \star \star}$ \\ ${ }^{1}$ Department of Mathematics, University of Torino, via C. Alberto 10, 10123 Torino, Italy \\ ${ }^{2}$ Lepage Research Institute, University of Prešov, 17 November 1, 08116 Prešov, Slovak Republic
}

\begin{abstract}
Classical Higgs fields and related canonical conserved quantities are defined by invariant variational problems on suitably defined gauge gluon bundles. We consider Lagrangian field theories which are assumed to be invariant with respect to the action of a gauge-natural group. As an illustrative example we exploit the 'gluon Lagrangian', i.e. a Yang-Mills Lagrangian on the $(1,1)$-order gauge-natural bundle of $S U(3)$-principal connections. The kernel of the gauge-natural Jacobi morphism for such a Lagrangian, by inducing a reductive split structure, canonically defines a 'gluon classical Higgs field'.
\end{abstract}

\section{Introduction}

In this note we shall deal with the definition of a classical Higgs field canonically induced by the invariance of a gluon Yang-Mills Lagrangian with respect to the gauge-natural infinitesimal tranformations of the bundle of $S U(3)$-connections, seen as a $(1,1)$-order gauge-natural affine bundle.

In particular, the Jacobi equations associated with the gluon Lagrangian define a canonical classical Higgs field, that is a reduction of the relevant principal bundle structure. It is noteworthy that the principal bundle structure is not the $S U(3)$-principal bundle, but its $(1,1)$-order gauge-natural prolongation. For basics on gauge-natural prolongations and applications in Physics, see e.g. [1, 2, 4]. Classical physical fields are sections of bundles functorially associated with gauge-natural prolongations of principal bundles, by means of left actions of Lie groups on manifolds.

Let us shortly summarize the geometric frame. Denote by $\boldsymbol{P} \rightarrow \boldsymbol{X}$ a principal bundle with structure group $\boldsymbol{G}, \operatorname{dim} \boldsymbol{X}=n$, by $L_{k}(\boldsymbol{X})$ the bundle of $k$-frames in $\boldsymbol{X}$. For $r \leq k$ the gauge-natural prolongation of $\boldsymbol{P}$ is $\boldsymbol{W}^{(r, k)} \boldsymbol{P} \doteq J_{r} \boldsymbol{P} \times_{\boldsymbol{X}} L_{k}(\boldsymbol{X})$, a principal bundle over $\boldsymbol{X}$ with structure group the semi-direct product $\boldsymbol{W}_{n}^{(r, k)} \boldsymbol{G} \equiv T_{n}^{r} \boldsymbol{G} \rtimes G L_{k}(n)$, with $G L_{k}(n)$ group of $k$-frames in $\mathbb{R}^{n}$ while $T_{n}^{r} \boldsymbol{G}$ is the space of $(r, n)$ velocities on $\boldsymbol{G}$.

Let $\boldsymbol{F}$ be a manifold and $\zeta: \boldsymbol{W}_{n}^{(r, k)} \boldsymbol{G} \times \boldsymbol{F} \rightarrow \boldsymbol{F}$ be a left action of $\boldsymbol{W}_{n}^{(r, k)} \boldsymbol{G}$ on $\boldsymbol{F}$. It is associated a gauge-natural bundle of order $(r, k)$ defined by $\boldsymbol{Y}_{\zeta} \doteq \boldsymbol{W}^{(r, k)} \boldsymbol{P} \times_{\zeta} \boldsymbol{F}$.

Since the group $\operatorname{Diff}(\boldsymbol{X})$ is not canonically embedded into the group $\operatorname{Aut}(\boldsymbol{P})$ of all automorphisms of the underling principal bundle $\boldsymbol{P}$, there is a priori no natural way of relating infinitesimal gauge transformations with infinitesimal base transformations. A canonical determination of Noether conserved quantities, without fixing any connection a priori, can be performed on a reduced subbundle of

\footnotetext{
$\star^{\star}$ e-mail: marcella.palese@unito.it

${ }^{\star}$ corresponding author, e-mail: ekkehart.winterroth@unito.it
} 
$W^{(r, k)} \boldsymbol{P}$ determined by the original $W_{n}^{(r, k)} \boldsymbol{G}$-invariant variational problem. Connections can be characterized by means of such a canonical reduction and conserved quantities can be characterized in terms of Higgs fields on gauge principal bundles having such a richer structure [6, 8, 9].

\section{Higgs field induced by 'gluon Lagrangians'}

As well known the Standard Model is a gauge theory with structure group $\boldsymbol{G}=S U(3) \times S U(2) \times U(1)$. One can consider the coupling with gravity by adding the principal spin bundle $\bar{\Sigma}$ with structure group Spin $(1,3)$; the structure bundle of the whole theory can be then taken to be the fibered product $\boldsymbol{\Sigma}=\bar{\Sigma} \times_{\boldsymbol{X}} \boldsymbol{P}$. There is an action of $\operatorname{Spin}(1,3)$ on a spinor matter manifold $V=\mathbb{C}^{k}$ and therefore a representation $\operatorname{Spin}(1,3) \times S U(3) \times S U(2) \times U(1) \times V$, given by a choice of Dirac matrices for each component of the spinor field.

A corresponding Lagrangian is therefore given by $\lambda=\bar{\psi}\left(i \gamma_{\mu} D^{\mu}-m\right) \psi-\frac{1}{4}\left(\mathcal{F}_{\mu \nu} \mathcal{F}^{\mu \nu}+\mathcal{F}_{\mu \nu}^{A} \mathcal{F}_{A}^{\mu \nu}+\right.$ $\mathcal{F}_{\mu \nu}^{a} \mathcal{F}_{a}^{\mu \nu}$ ). Experimental evidence concerned with symmetry properties of fundamental interactions shows the phenomenon of spontaneous symmetry breaking suggesting the presence of a scalar field called the Higgs boson on which the spin group acts trivially. A very clear and precise introduction to those topics can be found in [12].

For an illustrative purpose, let us then restrict to pure gluon fields assumed to be critical sections of the 'gluon Lagrangian' $\lambda_{\text {gluon }}=-\frac{1}{4} \mathcal{F}_{\mu \nu}^{a} \mathcal{F}_{a}^{\mu \nu}$. In this note, we shall therefore restrict to a principal bundle $\boldsymbol{\Sigma}$ with structure group $\boldsymbol{G}=S U(3)$ such that $\boldsymbol{\Sigma} / S U(3)=\boldsymbol{X}$ and $\operatorname{dim} \boldsymbol{X}=4$.

Recall that $W_{4}^{(1,1)} \boldsymbol{G}$ is the semi-direct product of $G L(4, \mathbb{R})$ on $T_{4}^{1} \boldsymbol{G}$, where $G L(4, \mathbb{R})$ is the structure group of linear frames in $\mathbb{R}^{4}$.

The set $\left\{j_{0}^{k} \alpha: \alpha: \mathbb{R}^{4} \rightarrow \mathbb{R}^{4}\right\}$, with $\alpha(0)=0$ locally invertible, equipped with the jet composition $j_{0}^{k} \alpha \circ j_{0}^{k} \alpha^{\prime}:=j_{0}^{k}\left(\alpha \circ \alpha^{\prime}\right)$ is a Lie group called the $k$-th differential group and denoted by $G_{4}^{k}$. For $k=1$ we have, of course, the identification $G_{4}^{1} \simeq G L(4, \mathbb{R})$. The principal bundle over $\boldsymbol{X}$ with group $G_{4}^{k}$ is called the $k$-th order frame bundle over $\boldsymbol{X}$ and will be denoted by $L_{k} \boldsymbol{X}$. For $k=1$ we have the identification $L_{1} \boldsymbol{X} \simeq L \boldsymbol{X}$, where $L \boldsymbol{X}$ is the usual (principal) bundle of linear frames over $\boldsymbol{X}$.

Unlike $J_{1} \Sigma, W^{(1,1)} \Sigma$ is a principal bundle over $\boldsymbol{X}$ with structure group $W_{4}^{(1,1)} \boldsymbol{G} \doteq T_{4}^{1} S U(3) \rtimes$ $G L(4, \mathbb{R}), T_{4}^{1} S U(3)$ being the Lie group of $(4,1)$-velocities of $S U(3)$ (if $u: \mathbb{R}^{4} \rightarrow S U(3)$, a generic element of $j_{0}^{1} u \in T_{4}^{1} S U(3)$ is represented by $g^{b}=u^{b}(0)$ and $\left.g_{v}^{b}=\left(\left.\partial_{v}\left(g^{-1} \cdot u(x)\right)\right|_{x=0}\right)^{b}\right)$. The group multiplication on $W^{(1,1)} \boldsymbol{G}$ being $\left(j_{0}^{1} \alpha, j_{0}^{1} a\right) \odot\left(j_{0}^{1} \beta, j_{0}^{1} b\right) \doteq\left(j_{0}^{1}(\alpha \circ \beta), j_{0}^{1}((a \circ \beta) \cdot b)\right)$ and denoting by $\cdot r$ the right action of $S U(3)$ on $\boldsymbol{\Sigma}$, the right action of $W_{4}^{(1,1)} \boldsymbol{G}$ on $W^{(1,1)} \boldsymbol{\Sigma}$ is then defined by $\left(j_{0}^{1} \rho, j_{x}^{1} \sigma\right) \odot$ $\left(j_{0}^{1} \alpha, j_{0}^{1} a\right) \doteq\left(j_{0}^{1}(\rho \circ \alpha), j_{x}^{1}\left(\sigma \cdot r\left(a \circ \alpha^{-1} \circ \rho^{-1}\right)\right)\right)$.

The bundle of principal connections on $\boldsymbol{\Sigma}$ is a gauge-natural bundle associated with the gaugenatural prolongation $W^{(1,1)} \boldsymbol{\Sigma}$. Consider the action $\zeta$ induced by the adjoint representation:

$\zeta: W_{4}^{1,1} \boldsymbol{G} \times\left(\mathbb{R}^{4}\right)^{*} \otimes \mathfrak{s u}(3) \rightarrow\left(\mathbb{R}^{4}\right)^{*} \otimes \mathfrak{s u}(3):\left(\left(g^{b}, g_{\mu}^{c}, \alpha_{\rho}^{\sigma}\right), f_{v}^{a}\right) \mapsto\left(A d_{g}\right)_{b}^{a}\left(f_{\sigma}^{b}-g_{\sigma}^{b}\right) \bar{\alpha}_{v}^{\sigma}$, where $\left(A d_{g}\right)_{b}^{a}$ are the coordinate expression of the adjoint representation of $\boldsymbol{G}=S U(3)$ and $g^{b}, g_{\mu}^{c}$ denote natural coordinates on $T_{4}^{1} S U(3)$. The sections of the associated bundle $C(\boldsymbol{\Sigma}) \doteq W^{(1,1)} \boldsymbol{\Sigma} \times{ }_{\zeta}\left(\mathbb{R}^{4}\right)^{*} \otimes \mathfrak{s u}(3) \rightarrow \boldsymbol{X}$ are in 1 to 1 correspondence with the principal connections on $\boldsymbol{\Sigma}$ and are called a $S U(3)$-connections. Clearly, by construction, $C(\boldsymbol{\Sigma})$ is a $(1,1)$-order gauge-natural affine bundle [4], see also [3].

Note that the Lie algebra of $W_{4}^{(1,1)} S U(3)$ is the semi-direct product of $\operatorname{gl}(4, \mathbb{R})$ with the Lie algebra, $\mathrm{t}_{4}^{1} \mathfrak{s u}(3)$, of $T_{4}^{1} S U(3)$. It is easy to characterize the semi-direct product of the two Lie algebras, from now on denoted by $\mathcal{S}$, as the direct $\operatorname{sum} \mathrm{t}_{4}^{1} \mathfrak{s u}(3) \oplus \mathfrak{g l}(4, \mathbb{R})$ with a bracket induced by the right action of $G L(4, \mathbb{R})$ on $T_{4}^{1} S U(3)$ given by the jet composition, in particular by the induced Lie algebra homomorphism $\mathrm{t}_{4}^{1} \mathfrak{s u}(3) \rightarrow \operatorname{hom}(\mathfrak{g l}(4, \mathbb{R}))$; given a base of $\mathrm{t}_{4}^{1} \mathfrak{s u}(3) \rtimes \mathfrak{g l}(4, \mathbb{R})$; the adjoint representation of the Lie group $W_{4}^{(1,1)} S U(3)$ is also easily defined (see e.g. $\left.[15,16], \S 1.3\right)$. Local coordinates on $W_{4}^{1,1} S U(3)$ are 
given by $\left(g^{b}, g_{\sigma}^{b} ; \alpha_{\sigma}^{\mu}\right)$ and induced local coordinates on $\mathcal{S}$ are given by $\left(Y^{a}, Y_{\mu}^{a}, X_{\sigma}^{\mu}\right)$. Local generators of the tangent space are of course partial derivative with respect to such local coordinates.

Consider the right action $R_{\hat{g}}: W^{(1,1)} \boldsymbol{\Sigma} \rightarrow W^{(1,1)} \boldsymbol{\Sigma}, \hat{g} \in W_{4}^{(1,1)} S U(3)$. Let $\Xi$ be a right invariant vector field on $W^{(1,1)} \boldsymbol{\Sigma}$. In coordinates we have $\Xi=\xi^{\lambda}(x) \partial_{\lambda}+\Xi^{a}(x) \tilde{\mathfrak{b}}_{a}$ where $\left(\tilde{\mathfrak{b}}_{a}\right)$ is the base of vertical right invariant vector fields on $W^{(1,1)} \boldsymbol{\Sigma}$ which are induced by the base $\left(\mathfrak{b}_{a}\right)$ of $\mathcal{S}$. They are sections of the bundle $T W^{(1,1)} \boldsymbol{\Sigma} / W_{4}^{(1,1)} S U(3) \rightarrow X$. We have $\tilde{\mathrm{b}}_{a}=\left(R_{\hat{g}}\right)_{a}^{b} \partial_{b}$, where the invertible matrix $\left(R_{\hat{g}}\right)_{a}^{b}$ is the matrix representation of $T R_{\hat{g}}$. It is clear that so-called Gell-Mann matrices $\lambda_{a}$ are matrix representations of $\mathfrak{b}_{a}$ and they therefore induce $\tilde{\mathfrak{b}}_{a}$ in the standard way. Analogously a matrix representation can be obtained for $\mathrm{b}_{a}^{\mu}$, being essentially $T_{4}^{1} S U(3) \rtimes G L(4, \mathbb{R}) \simeq\left(S U(3) \times\left(\mathbb{R}^{4}\right)^{*} \otimes \mathfrak{s} \mathfrak{u}(3)\right) \rtimes$ $G L(4, \mathbb{R})$.

\subsection{A variationally featured classical Higgs field associated with the gauge-natural invariant gluon Lagrangian}

The functorial linearity properties of the gauge-natural lift $\hat{\Xi}$ of infinitesimal automorphisms of $W^{(1,1)} \boldsymbol{\Sigma}$ to he bundle $C(\boldsymbol{\Sigma})$ of $S U(3)$-connections enable to define a gauge-natural generalized Jacobi morphism associated with a Lagrangian $\lambda$ and the variation vector field $\hat{\Xi}_{V}$, the vertical part of $\hat{\Xi}$, i.e. the linear morphism $\left.\left.\mathcal{J}\left(\lambda_{\text {gluon }}, \hat{\Xi}_{V}\right) \doteq \hat{\Xi}_{V}\right\rfloor \mathcal{E}\left(\hat{\Xi}_{V}\right\rfloor \mathcal{E}\left(\lambda_{\text {gluon }}\right)\right)$, where $\mathcal{E}$ is the Euler-Lagrange operator [5, 7]. Lifted tangent vector fields the vertical part of which are in the kernel $\Omega \doteq \operatorname{ker} \mathcal{J}\left(\lambda_{\text {gluon }}, \hat{\Xi}_{V}\right)$ are called generalized Jacobi vector fields and generate canonical covariant conserved quantities [8]. They have the property that the Lie derivative of critical sections of Yang-Mills Euler-Lagrange equations taken with respect to them is zero, i.e. they leave invariant the equations and their solutions. It is a subalgebra of the Lie algebra of vertical tangent vector field, which can be interpreted as internal symmetry algebra (see the Remark below). An explicit description of $\Omega$ for $\lambda_{\text {gluon }}$ and the corresponding Jacobi quadratic form $\mathcal{J}$ will appear in a separate paper.

A split structure given by $\mathfrak{R} \oplus \operatorname{Im} \mathcal{J}$ is defined on $V W^{(1,1)} \boldsymbol{\Sigma} / W_{4}^{(1,1)} S U(3)$. Furthermore, let $\mathfrak{h}$ be the Lie algebra of right-invariant vector fields on $W^{(1,1)} \boldsymbol{\Sigma}$ and $\mathfrak{f}$ the Lie subalgebra of generalized Jacobi vector fields; it is easy to see that the split structure is also reductive, being [ $[\mathfrak{f}, \operatorname{Im} \mathcal{J}]=\operatorname{Im} \mathcal{J}[5,7,8]$.

For each $\boldsymbol{p} \in W^{(1,1)} \boldsymbol{\Sigma}$, denote $\mathcal{S} \doteq \mathfrak{h}_{p}, \mathcal{R} \doteq \mathfrak{f}_{p}$ and $\mathcal{V} \doteq \operatorname{Im}_{\boldsymbol{j}}$; we have a reductive Lie algebra decomposition $\mathcal{S} \doteq \mathrm{t}_{4}^{1} \mathfrak{s u}(3) \rtimes \mathfrak{g l}(4, \mathbb{R})=\mathcal{R} \oplus \mathcal{V}$, with $[\mathcal{R}, \mathcal{V}]=\mathcal{V}$; we recall that $\mathcal{S}$ is the Lie algebra of the Lie group $W_{4}^{(1,1)} S U(3)$. $\mathcal{R}$ is a reductive Lie subalgebra of $\mathrm{t}_{4}^{1} \mathfrak{s u}(3) \rtimes \mathfrak{g l}(4, \mathbb{R})$. The reductive split structure is responsible of the canonical variational breaking of the symmetry group $W_{4}^{(1,1)} S U(3)$ and of the generation of Higgs fields. There exists an isomorphism between $\mathcal{V} \doteq \operatorname{Im} \mathcal{J}_{p}$ and $T_{x} X$ so that $\mathcal{V}$ turns out to be the image of an horizontal subspace. We caracterize a principal bundle $\boldsymbol{H} \rightarrow \boldsymbol{X}$, with $\operatorname{dim} \boldsymbol{H}=\operatorname{dim} \mathcal{S}$ and such that $\boldsymbol{X}=\boldsymbol{H} / \boldsymbol{R}$, where $\boldsymbol{R}$ is a Lie group of the Lie algebra $\mathcal{R}$ which is a closed subgroup of $W_{4}^{(1,1)} S U(3)$. The principal subbundle $\boldsymbol{H} \subset \boldsymbol{W}^{(1,1)} \boldsymbol{\Sigma}$ is then a reduced principal bundle [9].

We have the composite fiber bundle $W^{(1,1)} \boldsymbol{\Sigma} \rightarrow W^{(1,1)} \boldsymbol{\Sigma} / \boldsymbol{R} \rightarrow \boldsymbol{X}$, where $W^{(1,1)} \boldsymbol{\Sigma} / \boldsymbol{R}=$ $W^{(1,1)} \boldsymbol{\Sigma} \times_{W_{4}^{(1,1)} S U(3)} W_{4}^{(1,1)} S U(3) / \boldsymbol{R} \rightarrow \boldsymbol{X}$ is a gauge-natural bundle functorially associated with $W^{(1,1)} \boldsymbol{\Sigma} \times W_{4}^{(1,1)} S U(3) / \boldsymbol{R} \rightarrow \boldsymbol{X}$ by the right action of $W_{4}^{(1,1)} S U(3)$. The left action of $W_{4}^{(1,1)} S U(3)$ on $W_{4}^{(1,1)} S U(3) / \boldsymbol{R}$ is defined by the reductive Lie algebra decomposition.

According to [13, 14], we call a global section $h: X \rightarrow W^{(1,1)} \boldsymbol{\Sigma} / \boldsymbol{R}$ a gluon classical Higgs field.

The following Remark is now noteworthy .

Let $\omega$ be a principal connection on $W^{(1,1)} \boldsymbol{\Sigma}$; $\bar{\omega}$ principal connection on the principal bundle $\boldsymbol{H}$ defines the splitting $T_{\boldsymbol{p}} \boldsymbol{H} \simeq_{\bar{\omega}} \mathcal{R} \oplus \overline{\mathcal{H}}_{\boldsymbol{p}}, \boldsymbol{p} \in \boldsymbol{H}$. Note that, for each $\boldsymbol{q} \in W^{(1,1)} \boldsymbol{\Sigma}, T_{\boldsymbol{q}} W^{(1,1)} \boldsymbol{\Sigma} \simeq_{\omega}$ 
$V_{\boldsymbol{q}} W^{(1,1)} \boldsymbol{\Sigma} \oplus \mathcal{H}_{\boldsymbol{q}}$. We find that $V_{\boldsymbol{q}} W^{(1,1)} \boldsymbol{\Sigma} \simeq T_{\boldsymbol{q}} \boldsymbol{H} \simeq_{\bar{\omega}} \mathcal{R} \oplus \overline{\mathcal{H}}_{\boldsymbol{q}}, \boldsymbol{q} \in \boldsymbol{H}$, i.e. it is defined a Cartan connection $\hat{\omega}$ of type $\mathcal{S} / \mathcal{R}$, such that $\left.\hat{\omega}\right|_{V \boldsymbol{H}}=\bar{\omega}$. It is a connection on $W^{(1,1)} \boldsymbol{\Sigma}=\boldsymbol{H} \times_{\boldsymbol{R}} W_{4}^{(1,1)} S U(3) \rightarrow \boldsymbol{X}$, thus a Cartan connection on $\boldsymbol{H} \rightarrow \boldsymbol{X}$ with values in $\mathcal{S}$, the Lie algebra of the gauge-natural structure group of the theory; it splits into the $\mathcal{R}$-component which is a principal connection form on the $\mathcal{R}$-manifold $\boldsymbol{H}$, and the $\mathcal{V}$-component which is a displacement form.

A gluon gauge-natural Higgs field is therefore a global section of the Cartan horizontal bundle $\hat{\mathcal{H}}_{p}$, with $\boldsymbol{p} \in \boldsymbol{H}$, it is related with the displacement form defined by the $\mathcal{V}$-component of the Cartan connection $\hat{\omega}$ above. A global section $h$ of $W^{(1,1)} \boldsymbol{\Sigma} / \boldsymbol{R} \rightarrow \boldsymbol{X}$ defines a vertical covariant differential and therefore the Lie derivative of fields is constrained and it is parametrized by gluon Higgs fields $h$ characterized by $\Re[10,11]$.

\section{References}

[1] D.J. Eck: Gauge-natural bundles and generalized gauge theories, Mem. Amer. Math. Soc. 247 (1981) 1-48.

[2] L. Fatibene, M. Francaviglia: Natural and gauge natural formalism for classical field theories. A geometric perspective including spinors and gauge theories; Kluwer Academic Publishers, Dordrecht, 2003.

[3] M. Godina, P. Matteucci: Reductive G-structures and Lie derivatives, J. Geom. Phys. 47 (1) (2003) 66-86.

[4] I. Kolář, P.W. Michor, J. Slovák: Natural Operations in Differential Geometry, (Springer-Verlag, N.Y., 1993).

[5] M. Palese, E. Winterroth: Global Generalized Bianchi Identities for Invariant Variational Problems on Gauge-natural Bundles, Arch. Math. (Brno) 41 (3) (2005) 289-310.

[6] M. Palese, E. Winterroth: Covariant gauge-natural conservation laws, Rep. Math. Phys. 54 (3) (2004) 349-364.

[7] M. Palese, E. Winterroth: The relation between the Jacobi morphism and the Hessian in gaugenatural field theories, Theoret. Math. Phys. 152 (2) (2007) 1191-1200.

[8] M. Palese, E. Winterroth: Lagrangian reductive structures on gauge-natural bundles, Rep. Math. Phys. 62 (2) (2008) 229-239.

[9] M. Palese, E. Winterroth: Invariant variational problems and Cartan connections on gauge-natural bundles, AIP Conf. Proc. 1191 (2009) 160-165.

[10] M. Palese, E. Winterroth: A variational perspective on classical Higgs fields in gauge-natural theories, Theor. Math. Phys. 168 (1) (2011) 1002-1008.

[11] M. Palese, E. Winterroth: Higgs fields on spinor gauge-natural bundles, Journal of Physics: Conference Series 411 (2013) 012025.

[12] R.D. Peccei: Exact and Broken Symmetries in Particle Physics, arXiv:hep-ph/0002225.

[13] G. Sardanashvily: Geometry of classical Higgs fields, Int. J. Geom. Methods Mod. Phys. 3 (1) (2006) 139-148.

[14] G.A. Sardanashvily: Classical Higgs fields, Theoret. and Math. Phys. 181 (3) (2014) 1599-1611.

[15] J. Vondra: Natural prolongation of principal connections, PhD thesis, Masaryk University, Brno 2010.

[16] J. Janyška, J. Vondra: Natural principal connections on the principal gauge prolongation of a principal bundle, Rep. Math. Phys. 64 (3) (2009) 395-415. 